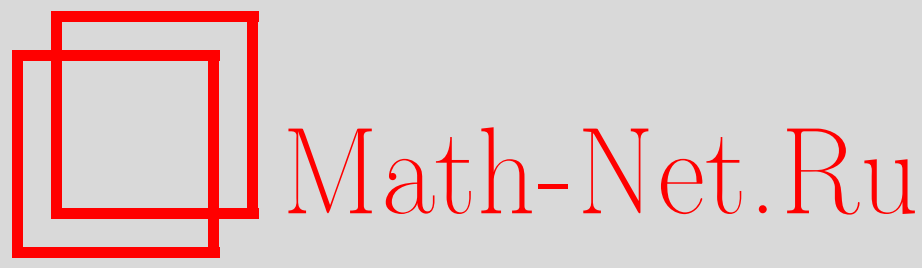

В. Б. Бобров, С. А. Тригер, Критическая опалесценция и состояние истинного диэлектрика в кулоновской системе, ТМФ, 2015, том 183, номер 1, 120-137

DOI: https://doi.org/10.4213/tmf8739

Использование Общероссийского математического портала Math-Net.Ru подразумевает, что вы прочитали и согласны с пользовательским соглашением http://www . mathnet.ru/rus/agreement

Параметры загрузки:

IP: 34.227 .88 .159

26 апреля 2023 г., 16:44:12

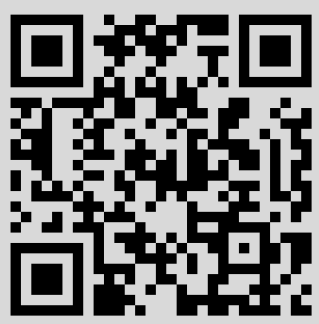




\title{
КРИТИЧЕСКАЯ ОПАЛЕСЦЕНЦИЯ И СОСТОЯНИЕ ИСТИННОГО ДИЭЛЕКТРИКА В КУЛОНОВСКОЙ СИСТЕМЕ
}

\begin{abstract}
На основе предельных соотношений для статических структурных факторов и анализа особенностей диэлектрической проницаемости рассмотрено явление критической опалесценции в двухкомпонентной кулоновской системе, состоящей из электронов и ядер одного сорта. Показано, что явление критической опалесценции может наблюдаться не только в критической точке, отвечающей фазовому переходу газ-жидкость, но и в окрестности состояния истинного диэлектрика с нулевой статической проводимостью. С учетом имеющихся экспериментальных данных можно предположить, что состояние истинного диэлектрика является предельным состоянием фазового перехода жидкость-жидкость, который сопровождается резким изменением электропроводности вещества. Установлено, что явление критической опалесценции при термодинамических параметрах, отвечающих состоянию истинного диэлектрика, может быть обусловлено обращением в бесконечность квадрата флуктуаций полного числа электронов и ядер в двухкомпонентной кулоновской системе, как это имеет место в критической точке, отвечающей фазовому переходу газ-жидкость.
\end{abstract}

Ключевые слова: критическая опалесценция, кулоновская система, статический структурный фактор, диэлектрическая проницаемость, истинный диэлектрик, равновесные функции Грина.

DOI: $10.4213 / \operatorname{tmf8739}$

\section{1. ВВЕДЕНИЕ}

До настоящего времени исследование явления критической опалесценции является одним из эффективных способов экспериментального определения критических параметров веществ [1], [2]. Качественное объяснение этого явления, которое проявляется в аномально сильном увеличении рассеяния света в узком интервале термодинамических параметров в окрестности критической точки индивидуальных веществ

* Объединенный институт высоких температур РАН, Москва, Россия.

E-mail: vic5907@mail.ru, satron@mail.ru

${ }^{\dagger}$ Национальный исследовательский университет "МЭИ", Москва, Россия

${ }^{\ddagger}$ Eindhoven University of Technology, Eindhoven, The Netherlands 
и растворов, было предложено Смолуховским [3]. Он предположил, что это явление есть следствие возрастания флуктуаций плотности в чистых (индивидуальных) веществах и флуктуаций концентраций в растворах в окрестности критической точки. Эту идею Смолуховского развил Эйнштейн [4], он использовал понятие диэлектрической проницаемости и учитывал ее зависимость от плотности вещества (или концентрации раствора). При этом Эйнштейн фактически осуществил современный метод вычисления флуктуации любой термодинамической величины, применив термодинамические соотношения и статистический принцип Больцмана, связывающий энтропию с вероятностью состояния замкнутой системы (см., например, [5]).

Дальнейший прогресс в теоретическом описании критической опалесценции связан с работой Орнштейна и Цернике [6], которые сформулировали локальный подход, в основе которого лежит предположение о том, что макроскопические параметры вещества в некоторой точке пространства зависят только от распределения частиц вещества в некоторой окрестности этой точки. Эту окрестность принято называть корреляционной сферой, а ее радиус - корреляционным радиусом или длиной корреляций (см., например, [7]). Идея этого подхода связана с тем, что структура вещества определяется радиальной функцией распределения $g(r)$, которая непосредственно связана со статическим структурным фактором $S(q)$, который согласно идее Эйнштейна определяет сечение рассеяния света,

$$
S(q)=1+n \int d \mathbf{r} e^{i \mathbf{q} \cdot \mathbf{r}}(g(r)-1) .
$$

Здесь $n=N / V$ - средняя плотность числа частиц, $N$ - полное число частиц в объеме $V$.

Такое рассмотрение относится к так называемой модели простой жидкости - однокомпонентной системы нейтральных частиц (атомов), взаимодействующих между собой посредством парного короткодействующего потенциала. Для модели простой жидкости справедливо предельное соотношение

$$
\lim _{q \rightarrow 0} S(q)=n k_{\mathrm{B}} T K_{T}, \quad K_{T}=-\frac{1}{V}\left(\frac{\partial V}{\partial P}\right)_{T, N},
$$

где $K_{T}$ - изотермическая сжимаемость рассматриваемой системы при температуpe $T$ и давлении $P$ в объеме $V, k_{\text {в }}$ - постоянная Больцмана. Далее учитывается, что фактическим определением критической точки фазового перехода газ-жидкость является обращение сжимаемости в бесконечность,

$$
K_{T} \rightarrow \infty
$$

Таким образом, согласно (2), (3) критическая опалесценция обусловлена расходимостью пространственного интеграла от радиальной функции распределения при критических значениях термодинамических параметров (см., например, [7]). Согласно теории Орнштейна-Цернике (ОЦ) [6] подобная расходимость обусловлена наличием так называемого "длинного хвоста" на больших расстояниях между атомами, т.е. в критической точке простая жидкость характеризуется корреляциями 
с бесконечным радиусом, даже если потенциал взаимодействия имеет конечный радиус. При появлении в теории подобной расходимости необходимо провести рассмотрение другой величины, которая связана с рассматриваемой, но ведет себя в интересующей области параметров регулярно. В теории ОЦ в качестве такой величины использована прямая корреляционная функция $c(r)$, которая связана с радиальной функцией распределения уравнением ОЦ

$$
\nu(r)=c(r)+n \int d \mathbf{r}_{1} \nu\left(\left|\mathbf{r}-\mathbf{r}_{1}\right|\right) c\left(r_{1}\right), \quad \nu(r)=g(r)-1 .
$$

Основное допущение Орнштейна и Цернике при рассмотрении критической опалесценции заключается в том, что фурье-образ прямой корреляционной функции $c(q)$ является четной аналитической функцией волнового вектора в длинноволновом пределе $(q \rightarrow 0)$ при любых термодинамических параметрах, включая критические параметры вещества. Прямым следствием этого допущения является так называемая лоренцева форма распределения интенсивности рассеянного света. Такое предсказание хорошо подтверждается для некоторых веществ, например для аргона [8]. При этом корреляционная длина стремится к бесконечности в критической точке. Однако в рамках теории ОЦ так называемые критические индексы (параметры, характеризующие степень расходимости свойств системы в окрестности критической точки) довольно сильно отличаются от соответствующих экспериментальных значений [9]. Длительное время это обстоятельство не вызывало удивления, так как считалось, что уравнение ОЦ (4) является всего лишь определением прямой корреляционной функции (более подробно см. [10], [11]). Подчеркнем, что теория ОЦ [6] имеет место только в рамках классической статистической механики.

С другой стороны, Ландау [12] высказал предположение о том, что явление критической опалесценции должно наблюдаться не только в критической точке, но и при фазовых переходах второго рода, и построил феноменологическую теорию, основанную на понятии параметра порядка. И хотя эксперимент Гугенхейма [13] не подтвердил гипотезу Ландау об аналитическом поведении свободной энергии как функции параметра порядка, феноменологический подход к рассмотрению критических явлений приобрел широкое распространение и, в частности, привел к введению понятия топологического параметра порядка для квантовых систем [14], [15].

При этом статистическое описание вещества сосредоточилось на изучении модельных систем (моделей). Эти системы, сконструированные теоретически, должны обладать двумя свойствами: а) они должны быть достаточно простыми, чтобы для них можно было получить точное решение или по крайней мере очень хорошее приближение; б) простота моделей не должна означать ее тривиальности [7]. Толчком к исследованию моделей явилась работа Онсагера [16], в которой было получено точное решение двумерной проблемы Изинга [17] для квадратной решетки с взаимодействием ближайших соседей в отсутствие магнитного поля и показано, что в термодинамическом пределе действительно происходит фазовый переход. Значения критических показателей для модели Изинга и для реальных систем в рамках "классических" теорий, подобных теории ОЦ, неверны. Поэтому были предприняты попытки теоретического исследования этой проблемы, которые в итоге привели к теории критических явлений Видома-Каданова-Вильсона (теория ВКВ) [18]-[20]. 
Эта теория основана на гипотезе подобия (скейлинга), гипотезе универсальности и уравнениях ренормализационной группы. Однако в теории ВКВ использовано множество непроверяемых приближений. Законы подобия, выведенные Вильсоном, могут и не быть всеобщими, хотя большой класс моделей и реальных систем, по-видимому, действительно подчиняется им [7].

Необходимо отметить, что теория ВКВ сосредоточена на исследовании фазовых переходов второго рода и на рассмотрении моделей для твердого тела (модель Изинга и иные модели, прежде всего модели ферромагнетиков). Применение этих результатов к рассмотрению критической точки фазового перехода газ-жидкость фактически основано на гипотезе универсальности критических явлений. Кроме того, теория ВKВ, являясь по своей сути феноменологической, не позволяет непосредственно описать явление критической опалесценции (см. (1), (2)). Тем не менее эта теория получила широкое распространение при описании критических явлений (см. [21]-[23] и цитированную там литературу).

Это привело, в свою очередь, к парадоксальной ситуации в теории критической точки фазового перехода газ-жидкость, которая является выделенной (особой) точкой обширной области термодинамических параметров, отвечающих неупорядоченному состоянию вещества. При этом всюду, за исключением окрестности критических параметров (фактическая область применения теории ВКВ), равновесные свойства веществ достаточно успешно описываются теорией простых (нейтральных) жидкостей, основанной на использовании интегральных уравнений для корреляционных функций. В то же время уравнение ОЦ (4), как уже было отмечено выше, позволяет в принципе дать микроскопическое обоснование явления критической опалесценции, хотя длительное время считалось невозможным дать описание критической точки перехода газ-жидкость на основе интегральных уравнений теории простой жидкости (см., например, [24]).

Однако попытки построения единой теории простой жидкости, включающей критическую точку перехода газ-жидкость, на основе уравнения ОЦ достигли определенного успеха. В работах [25], [26] (см. также [27]) развит микроскопический подход, основанный только на интегральных уравнениях теории жидкостей. В этих работах показано, что у уравнения ОЦ может быть два типа решения в зависимости от выбора второго уравнения, связывающего радиальную функцию распределения и прямую корреляционную функцию: обычное аналитическое (регулярное) решение и неаналитическое (критическое) решение.

Аналитическое решение отвечает всем стандартным результатам теории простой жидкости. Критическое решение приводит к результатам теории ВКВ критических явлений и некоторым новым результатам [25], [26]. Тем самым теория простых классических жидкостей позволяет в рамках традиционного моделирования вещества описать всю область термодинамических параметров, отвечающих неупорядоченному состоянию.

Однако Фишер [28] при анализе критических параметров для электролитов обратил внимание на существенные проблемы, касающиеся описания критической точки для систем с кулоновским взаимодействием. Для таких систем положения теории BКВ вызывают существенные сомнения, связанные с дальнодействующим характе- 
ром кулоновского взаимодействия. При этом классическая теория Дебая-Хюккеля, описывающая эффекты экранирования кулоновского взаимодействия в пределе низкой плотности, неприменима для термодинамических параметров, отвечающих критическим параметрам реальных кулоновских жидкостей. В частности, при исследовании критической точки так называемых "газообразных" металлов теория ВКВ практически не используется, как и результаты теории простой классической жидкости (см., например, [29]). Таким образом, традиционные теории критической опалесценции, рассмотренные выше, не позволяют дать описание этого явления для систем с кулоновским взаимодействием.

Вместе с тем в широкой области термодинамических параметров реальное вещество может быть адекватно представлено моделью нерелятивистских электронов (величины с индексом е) и ядер (величины с индексом с), взаимодействующих по закону Кулона. При этом с точки зрения термодинамической устойчивости кулоновских систем (KC) принципиальное значение имеют квантовые эффекты (см., например, [30]). Учитывая, что рассеяние света в КС определяется статическим структурным фактором электронов $S_{\text {eе }}(q)$ (см., например, [31]), для описания критической опалесценции в веществе необходимо доказать, что для величины $S_{\text {eе }}(q)$ справедливо соотношение, аналогичное предельному равенству (2). Эта задача решена в работе [32] на основе точных предельных соотношений для корреляционных функций в длинноволновом пределе для квантовой двухкомпонентной КС, соответствующей веществу, состоящему из одного химического элемента (ядер одного сорта) [33]-[35]. Соответствующее рассмотрение проводится с применением методов квантовой теории поля (диаграммной техники теории возмущений) с учетом особенностей использования большого канонического распределения Гиббса для КС [36].

При описании вещества как КС получает разрешение другой аспект изучения критической опалесценции, которому длительное время не уделялось достаточного внимания. Дело в том, что качественные идеи Смолуховского и Эйнштейна о рассеянии света на флуктуациях плотности нейтральных частиц простой жидкости не соответствуют современным представлениям о микроскопическом взаимодействии излучения с заряженными частицами. Для того чтобы использовать модель нейтральных атомов для объяснения явления критической опалесценции, фактически имеется единственная возможность - рассматривать атом как ядро с локализованными на нем электронами (аналогично традиционному квантово-механическому рассмотрению [37]), т. е. как составную частицу. Это очевидное на первый взгляд утверждение до настоящего времени не имеет строгого теоретического обоснования в связи с необходимостью учета тождественности электронов, а тем самым статистического описания самого атома в системе взаимодействующих электронов и ядер (подробнее см. [38]). Тем не менее в рамках модели нейтральных атомов связь между структурным фактором атомов $S(q)(1),(2)$ (строго говоря, ядер, т. е. $S_{\text {сс }}(q)$ ) и структурным фактором электронов $S_{\text {eе }}(q)$ принято устанавливать путем введения в рассмотрение формфактора атома (см., например, [39], [40]). Между тем в рамках последовательного рассмотрения корреляционных функций для квазинейтральной двухкомпонентной КС можно показать справедливость равенства [41]

$$
S_{\mathrm{ee}}(q \rightarrow 0)=Z_{\mathrm{c}} S_{\mathrm{cc}}(q \rightarrow 0),
$$


где $Z_{\text {с }}$ - заряд ядра. Казалось бы, можно считать, что соотношение (5) дает обоснование возможности применения результатов теории простой (нейтральной) жидкости для описания критической опалесценции (см. (1), (2)) в предположении, что вещество при определенных термодинамических параметрах можно рассматривать как квазиклассическую систему взаимодействующих атомов, представляемых как ядра с локализованными на них электронными состояниями. Однако такое рассмотрение не может быть использовано для проводников.

Учтем далее, что для всех известных веществ статическая проводимость $\sigma_{\text {st }}$ отлична от нуля при ненулевой температуре. Разделение веществ на диэлектрики и проводники носит условный характер и связано с тем, что величина статической проводимости у диэлектриков значительно меньше, чем у проводников. В этом смысле строгое разделение веществ на проводники и диэлектрики можно дать только при нулевой температуре (см., например, [42]-[44]). Таким образом, понятие истинного диэлектрика $\left(\sigma_{\mathrm{st}} \rightarrow 0\right)$ как состояния вещества с нулевой статической проводимостью является предельным, как, например, понятие критической точки. С этой точки зрения, учитывая многочисленные экспериментальные данные о достаточно резком изменении проводимости при определенных термодинамических параметрах, возникает вопрос о возможности фазового перехода металл-диэлектрик при ненулевых температурах и соответствующих критических явлениях (подробнее см. [45]-[47]). Если принять, что переход металл-диэлектрик является фазовым переходом первого рода, то в соответствующей критической точке вещество находится в состоянии истинного диэлектрика [48]-[50]. Таким образом, в чистом веществе, состоящем из одного химического элемента, помимо “обычной” критической точки может существовать вторая критическая точка, отвечающая состоянию истинного диэлектрика [51].

В настоящей работе детально рассмотрены предельные соотношения для статических структурных факторов и проведен анализ особенностей диэлектрической проницаемости. На этой основе установлено, что явление критической опалесценции при термодинамических параметрах, отвечающих состоянию истинного диэлектрика, может быть обусловлено обращением в бесконечность квадрата флуктуаций полного числа электронов и ядер в двухкомпонентной КС, как это имеет место в критической точке, отвечающей фазовому переходу газ-жидкость в неупорядоченной системе атомов.

\section{2. ПРЕДЕЛЬНЫЕ СООТНОШЕНИЯ ДЛЯ СТРУКТУРНЫХ ФАКТОРОВ И ИЗОТЕРМИЧЕСКАЯ СЖИМАЕМОСТЬ}

Рассмотрим равновесную двухкомпонентную $\mathrm{KC}$, состоящую из электронов и ядер одного сорта, при температуре $T$ в объеме $V$. При этом, как обычно, для квантовых систем вычисление равновесных свойств осуществляется с использованием большого канонического распределения Гиббса и методов квантовой теории поля (диаграммной техники теории возмущений) с учетом выполнения условия квазинейтральности [30]

$$
\sum_{a=\mathrm{e}, \mathrm{c}} Z_{a} e n_{a}=0 .
$$


Здесь $n_{a}=\left\langle\widehat{N}_{a}\right\rangle / V$ - средняя плотность числа частиц сорта $a$, которые характеризуются массой $m_{a}$, зарядом $Z_{a} e$ и спином $s_{a}, \widehat{N}_{a}$ - оператор полного числа частиц сорта $a$ в объеме $V$, угловые скобки $\langle\cdot\rangle$ обозначают усреднение с большим каноническим распределением Гиббса, которое задается в рассматриваемой двухкомпонентной $\mathrm{KC}$ химическими потенциалами $\mu_{\mathrm{e}}$ для электронов и $\mu_{\mathrm{c}}$ для ядер.

Специфика $\mathrm{KC}$ проявляется в том, что химические потенциалы частиц $\mu_{a}$, как и средние числа частиц $\left\langle\widehat{N}_{a}\right\rangle$, считаются формально независимыми, а условие квазинейтральности (6) используется в окончательных выражениях для соответствующих физических величин. При этом выполняется равенство

$$
\left.u_{a b}(\mathbf{q})\right|_{\mathbf{q}=0}=0
$$

где $u_{a b}(\mathbf{q})$ - фурье-преобразование для кулоновского потенциала взаимодействия частиц сортов $a$ и $b$, которое при ненулевых волновых векторах имеет вид $u_{a b}(q)=$ $4 \pi Z_{a} Z_{b} e^{2} / q^{2}$ (подробнее см. [36]).

По аналогии с соотношением (1) в $\mathrm{KC} \mathrm{статический} \mathrm{структурный} \mathrm{фактор} S_{a b}(q)$ для частиц сортов $a$ и $b$ непосредственно связан с соответствующей радиальной функцией распределения $g_{a b}(r)$ общим соотношением

$$
S_{a b}(q)=\delta_{a, b}+\left(n_{a} n_{b}\right)^{1 / 2} \int d \mathbf{r} e^{i \mathbf{q} \cdot \mathbf{n}}\left(g_{a b}(r)-\delta_{a, b}\right),
$$

где $\delta_{a, b}$ - символ Кронекера. Для вычисления статического структурного фактора в квантовой $\mathrm{KC}$ используется связь величины $S_{a b}(q)$ с соответствующей запаздывающей функцией Грина “плотность-плотность” $\chi_{a b}^{R}(q, \omega)[31]$

$$
\begin{aligned}
\left(n_{a} n_{b}\right)^{1 / 2} S_{a b}(q) & \equiv V^{-1}\left\langle\delta \hat{\rho}_{\mathbf{q}}^{a}(0) \delta \hat{\rho}_{-\mathbf{q}}^{b}(0)\right\rangle= \\
& =-\frac{\hbar}{\pi} \int_{-\infty}^{\infty} d \omega \frac{\operatorname{Im} \chi_{a b}^{R}(q, \omega)}{1-e^{-\hbar \omega / k_{\mathrm{B}} T}}, \\
\chi_{a b}^{R}(q, z) & =-\frac{i}{\hbar V} \int_{0}^{\infty} d t e^{i z t}\left\langle\left[\delta \hat{\rho}_{\mathbf{q}}^{a}(t), \delta \hat{\rho}_{-\mathbf{q}}^{b}(0)\right]\right\rangle \equiv \\
& \equiv V^{-1}\left\langle\left\langle\delta \hat{\rho}_{\mathbf{q}}^{a}(0) \mid \delta \hat{\rho}_{-\mathbf{q}}^{b}(0)\right\rangle\right\rangle_{z} .
\end{aligned}
$$

Здесь $\delta \hat{\rho}_{\mathbf{q}}^{a}(t)$ - фурье-преобразование оператора $\delta \hat{n}_{a}(\mathbf{r}, t)=\hat{n}_{a}(\mathbf{r}, t)-n_{a}$ в представлении Гейзенберга, $\hat{n}_{a}(\mathbf{r})=\widehat{\Psi}_{a}^{+}(\mathbf{r}) \widehat{\Psi}_{a}(\mathbf{r}), \widehat{\Psi}_{a}^{+}(\mathbf{r})$ и $\widehat{\Psi}_{a}^{+}(\mathbf{r})$ - соответственно полевые операторы рождения и уничтожения частиц сорта $a$. Соотношения (9), (10) следует понимать в термодинамическом пределе

$$
V \rightarrow \infty, \quad\left\langle\widehat{N}_{a}\right\rangle \rightarrow \infty, \quad n_{a}=\frac{\left\langle\widehat{N}_{a}\right\rangle}{V}=\text { const }
$$

Согласно (10) функции Грина $\chi_{a b}^{R}(q, z)$ являются аналитическими функциями в верхней полуплоскости комплексных значений $z(\operatorname{Im} z>0)$. На мнимой оси в точках $\hbar z=i \Omega_{n}, \Omega_{n}=2 \pi n k_{\mathrm{B}} T$, где $n$ - целое число, включая нуль, запаздывающие функции Грина $\chi_{a b}^{R}(q, z)$ совпадают с соответствующими температурными 
функциями Грина $\chi_{a b}^{T}\left(q, i \Omega_{n}\right)$, для вычисления которых существуют хорошо разработанные методы квантовой теории поля (диаграммной техники теории возмущений) [52]. В частности, для функций Грина $\chi_{a b}^{T}\left(q, i \Omega_{n}\right)$ можно записать уравнение (см. [30]), связанное с выделением неприводимых по одной линии взаимодействия $u_{a b}(q)$ в " $q$-канале" частей, называемых парциальными поляризационными операторами $\Pi_{a b}\left(q, i \Omega_{n}\right)$ :

$$
\chi_{a b}^{T}\left(q, i \Omega_{n}\right)=\Pi_{a b}\left(q, i \Omega_{n}\right)+\sum_{d, f} \Pi_{a d}\left(q, i \Omega_{n}\right) u_{d f}(q) \chi_{f b}^{T}\left(q, i \Omega_{n}\right) .
$$

Для рассматриваемого случая двухкомпонентной КС из (11) следует

$$
\begin{aligned}
\chi_{\mathrm{ee}}^{T}\left(q, i \Omega_{n}\right) & =\varepsilon^{-1}\left(q, i \Omega_{n}\right)\left\{\Pi_{\mathrm{ee}}\left(q, i \Omega_{n}\right)-u_{\mathrm{cc}}(q)\left[\Pi_{\mathrm{ee}}\left(q, i \Omega_{n}\right) \Pi_{\mathrm{cc}}\left(q, i \Omega_{n}\right)-\Pi_{\mathrm{ec}}^{2}\left(q, i \Omega_{n}\right)\right]\right\}, \\
\chi_{\mathrm{cc}}^{T}\left(q, i \Omega_{n}\right) & =\varepsilon^{-1}\left(q, i \Omega_{n}\right)\left\{\Pi_{\mathrm{cc}}\left(q, i \Omega_{n}\right)-u_{\mathrm{ee}}(q)\left[\Pi_{\mathrm{ee}}\left(q, i \Omega_{n}\right) \Pi_{\mathrm{cc}}\left(q, i \Omega_{n}\right)-\Pi_{\mathrm{ec}}^{2}\left(q, i \Omega_{n}\right)\right]\right\}, \\
\chi_{\mathrm{ec}}^{T}\left(q, i \Omega_{n}\right) & =\chi_{\mathrm{ce}}^{T}\left(q, i \Omega_{n}\right)= \\
& =\varepsilon^{-1}\left(q, i \Omega_{n}\right)\left\{\Pi_{\mathrm{ec}}\left(q, i \Omega_{n}\right)+u_{\mathrm{ec}}(q)\left[\Pi_{\mathrm{ee}}\left(q, i \Omega_{n}\right) \Pi_{\mathrm{cc}}\left(q, i \Omega_{n}\right)-\Pi_{\mathrm{ec}}^{2}\left(q, i \Omega_{n}\right)\right]\right\},
\end{aligned}
$$

Здесь $\varepsilon\left(q, i \Omega_{n}\right)$ - диэлектрическая проницаемость $\mathrm{KC}$

$$
\varepsilon\left(q, i \Omega_{n}\right)=\mathbf{1}-u(q) \Pi_{Z Z}\left(q, i \Omega_{n}\right), \quad \Pi_{Z Z}\left(q, i \Omega_{n}\right)=\sum_{a, b} Z_{a} Z_{b} e^{2} \Pi_{a b}\left(q, i \Omega_{n}\right),
$$

$\Pi_{Z Z}\left(q, i \Omega_{n}\right)$ - поляризационный оператор "заряд-заряд", $u(q)=4 \pi / q^{2}$.

Далее с помощью спектральных представлений для запаздывающих и температурных функций Грина нетрудно убедиться [33], что вместо (9) для вычисления статического структурного фактора может быть использовано равенство

$$
\left(n_{a} n_{b}\right)^{1 / 2} S_{a b}(q)=-k_{\mathrm{B}} T \sum_{n=-\infty}^{\infty} \chi_{a b}^{T}\left(q, i \Omega_{n}\right)
$$

из которого непосредственно следует

$$
k_{\mathrm{B}} T \chi_{a b}^{T}(q, 0)+k_{\mathrm{B}} T \Phi_{a b}(q)=-\left(n_{a} n_{b}\right)^{1 / 2} S_{a b}(q), \quad \Phi_{a b}(q)=\sum_{n \neq 0} \chi_{a b}^{T}\left(q, i \Omega_{n}\right) .
$$

При этом функция $\Phi_{a b}(q)$ в области малых волновых векторов $q$ удовлетворяет условиям (подробнее см. [33]-[35])

$$
\Phi_{a b}(q) \sim q^{2}, \quad \lim _{q \rightarrow 0} \Phi_{a b}(q)=0,
$$

поэтому согласно (14) для любых равновесных систем выполняется предельное соотношение

$$
\left(n_{a} n_{b}\right)^{1 / 2} \lim _{q \rightarrow 0} S_{a b}(q)=-k_{\mathrm{B}} T \lim _{q \rightarrow 0} \chi_{a b}^{R}(q, 0) .
$$

Учтем теперь, что для поляризационных операторов нормальных $\mathrm{KC} \mathrm{имеет} \mathrm{место}$ равенство [34], [35]

$$
\lim _{q \rightarrow 0} \Pi_{a b}(q, 0)=-\Delta_{a b}, \quad \Delta_{a b}=\Delta_{b a} \equiv\left(\frac{\partial n_{a}}{\partial \mu_{b}}\right)_{T, \mu_{d} \neq \mu_{b}},
$$


где $n_{a}\left(T,\left\{\mu_{b}\right\}\right)$ - средняя плотность числа частиц сорта $a$ как функция температуры $T$ и химических потенциалов $\left\{\mu_{b}\right\}$ всех сортов заряженных частиц.

Подставляя (18) в (12), (13), находим

$$
\lim _{q \rightarrow 0} \chi_{\mathrm{cc}}^{R}(q, 0)=\left(\frac{1}{Z_{\mathrm{c}}}\right)^{2} \lim _{q \rightarrow 0} \chi_{\mathrm{ee}}^{R}(q, 0)=\frac{1}{Z_{\mathrm{c}}} \lim _{q \rightarrow 0} \chi_{\mathrm{ce}}^{R}(q, 0)=-n_{\mathrm{c}}^{2} \eta_{T},
$$

где величина $\eta_{T}=\eta_{T}\left(T,\left\{\mu_{b}\right\}\right)$ равна

$$
\eta_{T}=\frac{\Delta_{\mathrm{ee}} \Delta_{\mathrm{cc}}-\Delta_{\mathrm{ec}}^{2}}{n_{\mathrm{c}}^{2}\left(\Delta_{\mathrm{ee}}-2 Z_{\mathrm{c}} \Delta_{\mathrm{ec}}+Z_{\mathrm{c}}^{2} \Delta_{\mathrm{cc}}\right)} .
$$

В свою очередь, из (17), (19), (20) следует [34], [35]

$$
\lim _{q \rightarrow 0} S_{\mathrm{cc}}(q)=n_{\mathrm{c}} k_{\mathrm{B}} T \eta_{T}, \quad \lim _{q \rightarrow 0} S_{\mathrm{cc}}(q)=\frac{1}{Z_{\mathrm{c}}} \lim _{q \rightarrow 0} S_{\mathrm{ee}}(q)=\left(\frac{1}{Z_{\mathrm{c}}}\right)^{1 / 2} \lim _{q \rightarrow 0} S_{\mathrm{ec}}(q) .
$$

Согласно (21) приходим к выводу, что в длинноволновом пределе статический структурный фактор электронов $S_{\text {eе }}(q)$, измеряемый в экспериментах по рассеянию электромагнитного излучения, отличается от статического структурного фактора ядер $S_{\mathrm{cc}}(q)$, измеряемого в экспериментах по рассеянию нейтронов, на множитель, равный заряду ядра [41].

Чтобы установить соответствие с предельным соотношением (2), имеющим место в модели простой (нейтральной) жидкости, необходимо убедиться в том, что величина $\eta_{T}$ равна изотермической сжимаемости $K_{T}$ для $\mathrm{KC}$.

Большой термодинамический потенциал $\Omega\left(T, V,\left\{\mu_{\mathrm{c}}\right\}\right)$, отвечающий рассмотрению КС с использованием большого канонического распределения Гиббса, удовлетворяет соотношениям [36]

$$
\Omega\left(T, V,\left\{\mu_{a}\right\}\right)=-P\left(T,\left\{\mu_{a}\right\}\right) V, \quad d \Omega=-S d T-P d V-\sum_{a}\left\langle\widehat{N}_{a}\right\rangle d \mu_{a}
$$

где $S$ - энтропия КС. Из (22) непосредственно следует

$$
\left(\frac{\partial P}{\partial \mu_{a}}\right)_{T, \mu_{d} \neq \mu_{a}}=n_{a}\left(T,\left\{\mu_{b}\right\}\right)
$$

Перейдем к определению изотермической сжимаемости $\mathrm{KC} K_{T}$, которая с учетом (23) имеет вид

$$
K_{T}=-\frac{1}{V}\left(\frac{\partial V}{\partial P}\right)_{T,\left\{N_{a}\right\}}=\left\{\sum_{a, b} n_{a} n_{b}\left(\frac{\partial \mu_{a}}{\partial n_{b}}\right)_{n_{d}, T}\right\}^{-1} .
$$

Чтобы провести вычисления с использованием большого канонического распределения Гиббса, необходимо перейти от производных $\left(\partial \mu_{a} / \partial n_{b}\right)_{n_{d}, T}$ к производным $\left(\partial n_{a} / \partial \mu_{b}\right)_{\mu_{d}, T}$. Для этого воспользуемся термодинамическим равенством

$$
\sum_{d}\left(\frac{\partial \mu_{a}}{\partial n_{d}}\right)_{T, n_{f} \neq n_{d}}\left(\frac{\partial n_{d}}{\partial \mu_{b}}\right)_{T, \mu_{f} \neq \mu_{b}}=\delta_{a, b} .
$$


Для двухкомпонентной $\mathrm{KC}$ с помощью системы уравнений (25) нетрудно установить связь между производными $\left(\partial \mu_{a} / \partial n_{b}\right)_{n_{d}, T}$ и $\left(\partial n_{a} / \partial \mu_{b}\right)_{\mu_{d}, T}$. В результате для двухкомпонентной $\mathrm{KC}$, состоящей из электронов и ядер одного сорта, получаем

$$
K_{T}=\frac{\Delta_{\mathrm{ee}} \Delta_{\mathrm{cc}}-\Delta_{\mathrm{ec}}^{2}}{n_{\mathrm{c}}^{2} \Delta_{\mathrm{ee}}-2 n_{\mathrm{e}} n_{\mathrm{c}} \Delta_{\mathrm{ec}}+n_{\mathrm{e}}^{2} \Delta_{\mathrm{cc}}} .
$$

Учитывая, что изотермическая сжимаемость $K_{T}$ является физической величиной, мы можем использовать в соотношении (26) условие квазинейтральности (6). Сравнивая (20) и (26), приходим к выводу, что

$$
K_{T}=\eta_{T}
$$

Таким образом, в двухкомпонентной КС явление критической опалесценции связано со стремлением изотермической сжимаемости к бесконечности, аналогично существующим представлениям для простой (нейтральной) жидкости (см. (3)) [32]. Более того, согласно (21) это явление может быть обнаружено не только при рассеянии электромагнитного излучения, но и в экспериментах по рассеянию нейтронов.

\section{3. ОСОБЕННОСТИ ДИЭЛЕКТРИЧЕСКОЙ ПРОНИЦАЕМОСТИ И СОСТОЯНИЕ ИСТИННОГО ДИЭЛЕКТРИКА}

Прежде чем провести анализ выражения (26) для изотермической сжимаемости КС с точки зрения выполнения условия (3), рассмотрим величину диэлектрической проницаемости (ДП) $\varepsilon(q, \omega)$, которая определяет скалярное поле $U^{\text {tot }}(q, \omega)$ в среде при воздействии на нее слабого внешнего поля с потенциалом $U^{\mathrm{ext}}(q, \omega): U^{\mathrm{tot}}(q, \omega)=$ $U^{\operatorname{ext}}(q, \omega) / \varepsilon(q, \omega)$. При таком определении ДП $\varepsilon(q, \omega)$ совпадает с продольной ДП $\varepsilon^{\mathrm{l}}(q, \omega)$ в $\mathrm{KC}[30]$.

Особое значение имеют строгие соотношения для ДП, которые определяют общие закономерности ее поведения в зависимости от величины волнового вектора $q$ и частоты $\omega$. Одной из таких закономерностей является зависимость значений функции $\varepsilon(q, \omega)$ от порядка предельных переходов $\omega \rightarrow 0, q \rightarrow 0$ и $q \rightarrow 0, \omega \rightarrow 0$ [53]

$$
\lim _{q \rightarrow 0} \varepsilon(q) \neq \lim _{\omega \rightarrow 0} \varepsilon(\omega),
$$

где $\varepsilon(q)=\lim _{\omega \rightarrow 0} \varepsilon(q, \omega)$ - статическая ДП, характеризующая равновесное состояние $\mathrm{KC}$ в электростатическом внешнем поле, $\varepsilon(\omega)=\lim _{q \rightarrow 0} \varepsilon(q, \omega)$ - динамическая ДП, которая непосредственно связана с ДП КС $\sigma(\omega)$ в слабонеоднородном внешнем поле,

$$
\varepsilon(\omega)=1+\frac{4 \pi i}{\omega} \sigma(\omega) .
$$

При этом непосредственно измеряемая статическая проводимость $\sigma_{\text {st }}$ определяется соотношением [53]

$$
\sigma_{\mathrm{st}}=\lim _{\omega \rightarrow 0} \sigma(\omega) \geqslant 0 .
$$

Как уже было отмечено выше, для всех известных веществ статическая проводимость $\sigma_{\mathrm{st}}$ отлична от нуля при ненулевой температуре. Разделение веществ на

5 Теоретическая и математическая физика, т. 183, № 1, 2015 г. 
классы по величине статической проводимости (электропроводности) носит условный характер: проводники (высокая электропроводность), диэлектрики (низкая электропроводность) и промежуточный класс - полупроводники (промежуточная электропроводность, сильно зависящая от внешних условий). Таким образом, согласно $(29),(30)$ ДП $\varepsilon(\omega)$ любого вещества в статическом пределе $\omega \rightarrow 0$ имеет особенность [53]:

$$
\left.\varepsilon(\omega)\right|_{\omega \rightarrow 0} \rightarrow \frac{4 \pi i}{\omega} \sigma_{\text {st }} .
$$

В свою очередь, для статической ДП $\varepsilon(q)$ выполняется предельное соотношение [53]

$$
\lim _{q \rightarrow 0} q^{2}[\varepsilon(q)-1]=\varkappa^{2} \geqslant 0,
$$

где величина $\varkappa$ в соответствующих случаях совпадает с волновыми векторами Дебая и Томаса-Ферми. Учтем далее, что статическая ДП $\varepsilon(q)$ определяет соотношение между потенциалом полного электростатического поля в среде $U^{\text {tot }}(q)=$ $\lim _{\omega \rightarrow 0} U^{\text {tot }}(q, \omega)$ и потенциалом слабого внешнего поля $U^{\operatorname{ext}}(q)=\lim _{\omega \rightarrow 0} U^{\text {ext }}(q, \omega)$

$$
U^{\text {tot }}(q)=\frac{U^{\operatorname{ext}}(q)}{\varepsilon(q)}
$$

Как видно из (32), (33), величина $\varkappa^{-1}$ характеризует глубину проникновения электростатического поля в вещество. Таким образом, по аналогии с рассуждениями относительно значений статической проводимости $\sigma_{\mathrm{st}}$ (см. выше) можно утверждать, что в проводниках глубина проникновения очень мала, в то время как у диэлектриков она может быть одного порядка с макроскопическими параметрами системы [48], [49]. Подтверждение этому можно найти в книге [30], где для вычисления статической ДП $\varepsilon(q)$ развито обобщенное приближение хаотических фаз, позволяющее учесть связанные состояния электронов и ядер. Фактически речь идет о разделении состояний частиц в КС на "связанные" состояния и состояния "рассеяния", в которых заряженные частицы могут распространяться по всему объему КС.

Таким образом, неравенство (28) обусловлено наличием особенности $1 / \omega$ в статическом пределе $\omega \rightarrow 0$ у динамической ДП $\varepsilon(\omega)$ (см. (31)) и особенности $1 / q^{2}$ в длинноволновом пределе $q \rightarrow 0$ у статической ДП $\varepsilon(q)$ (см. (32)). Из приведенного выше анализа ясно, что обе эти особенности ДП $\varepsilon(q, \omega)$ связаны с состояниями "рассеяния" заряженных частиц в равновесной КС. Отметим, что основой для исследования истинного диэлектрика как состояния с нулевой статической проводимостью $\left(\sigma_{\mathrm{st}} \rightarrow 0\right)$ при рассмотрении перехода металл-диэлектрик является решение вопроса о соотношении "связанных" состояний и состояний "рассеяния" [45]-[47].

В связи с этим естественно предположить, что между указанными особенностями ДП $\varepsilon(q, \omega)$, приводящими к неравенству (28), существует взаимосвязь [48], [49]. Доказательство этого утверждения [50] основано на теории линейного отклика (см., например, [30]), позволяющей точно учесть взаимодействие заряженных частиц в КС. Согласно теории линейного отклика ДП $\varepsilon(q, \omega)$ однородной и изотропной $\mathrm{KC}$ определяется следующим соотношением при $q \neq 0$ :

$$
\varepsilon^{-1}(q, \omega)=1+\frac{4 \pi}{q^{2}} \chi_{Z Z}^{R}(q, \omega),
$$


где $\chi_{Z Z}^{R}(q, \omega)=\sum_{a b} Z_{a} Z_{b} e^{2} \chi_{a b}^{R}(q, \omega)$ - запаздывающая функция Грина “заряд-заряд” (см. (10)),

$$
\chi_{Z Z}^{R}(q, \omega)=\int_{0}^{\infty} d t e^{i \omega t} \varphi_{\chi}(q, t), \quad \varphi_{\chi}(q, t)=-\frac{i}{\hbar V}\left\langle\left[\delta \hat{\rho}_{\mathbf{q}}^{z}(t), \delta \hat{\rho}_{-\mathbf{q}}^{z}(0)\right]\right\rangle .
$$

Здесь $\delta \hat{\rho}_{\mathbf{q}}^{z}(t)=\sum_{a} Z_{a} e \delta \hat{\rho}_{\mathbf{q}}^{a}(t)$ - фурье-образ оператора флуктуации плотности заряда в представлении Гейзенберга. Определение (35) для временно́й корреляционной функции $\varphi_{\chi}(q, t)$ следует понимать в термодинамическом пределе.

Функция Грина $\chi_{Z Z}^{R}(q, \omega)$ является типичным примером обобщенной восприимчивости, которая определена при действительных частотах $\omega$ [54]. Поведение временно́й корреляционной функции $\varphi_{\chi}(q, t)$ при больших временах $(t \rightarrow \infty)$ должно обеспечивать сходимость интеграла в (35) при произвольных действительных частотах $\omega$, в частности

$$
\lim _{t \rightarrow \infty} \varphi_{\chi}(q, t)=0 .
$$

Исходя из определения (35) и условия (36) запаздывающая функция Грина $\chi_{Z Z}^{R}(q, \omega)$ может быть доопределена в верхней полуплоскости комплексных частот $z$, где величина $\chi_{Z Z}^{R}(q, z)$ является аналитической функцией. Но для вычисления запаздывающих функций Грина не существует регулярной процедуры. Однако, как было отмечено выше, на мнимой оси комплексных $z$ значения запаздывающих функций Грина совпадают со значениями соответствующих температурных функций Грина при $\hbar z=i \Omega_{n}, \Omega_{n}=2 \pi n T, n=0,1,2, \ldots$, для вычисления которых существует хорошо разработанная температурная диаграммная техника теории возмущений (см. (12)). В частности, для значений ДП $\varepsilon(q, z)$ в точках $\hbar z=i \Omega_{n}$ на мнимой оси справедливо соотношение (13).

После вычисления соответствующей температурной функции Грина осуществляется процедура аналитического продолжения с дискретного множества точек на мнимой оси $i \Omega_{n}$ на верхнюю полуплоскость комплексных $z$. В рамках такой процедуры может быть найдена интересующая нас функция в верхней полуплоскости комплексных частот $z$ [52]. Далее, для определения найденной таким образом величины как функции действительных частот $\omega$ используется следующий прием, который принято называть правилом обхода полюсов Ландау (см., например, [55]):

$$
\chi_{Z Z}^{R}(q, \omega)=\lim _{\delta \rightarrow+0} \chi_{Z Z}^{R}(q, \omega+i \delta) .
$$

Отметим, что соотношение (10) может быть использовано только после перехода к термодинамическому пределу [56].

Таким образом, с использованием диаграммной техники теории возмущений можно показать (см. (11)-(13)), что при $\operatorname{Im} z>0$

$$
\chi_{Z Z}^{R}(q, z)=\frac{\Pi_{Z Z}(q, z)}{\varepsilon(q, z)}, \quad \varepsilon(q, z)=1-u(q) \Pi_{Z Z}(q, z) .
$$

Следовательно, ДП $\varepsilon(q, \omega)$ однородной и изотропной $\mathrm{KC} \mathrm{при} \mathrm{действительных} \mathrm{часто-}$ тах $\omega$ может быть представлена в виде

$$
\varepsilon(q, \omega)=1-u(q) \Pi_{Z Z}(q, \omega), \quad \Pi_{Z Z}(q, \omega)=\lim _{\delta \rightarrow+0} \Pi_{Z Z}(q, \omega+i \delta) .
$$


При этом поляризационный оператор $\Pi_{Z Z}(q, z)$, в отличие от функции Грина $\chi_{Z Z}^{R}(q, z)$, не имеет особенностей в области малых волновых векторов $q$, связанных с видом кулоновского потенциала взаимодействия $u(q)$ [34], [35]. Благодаря этому обстоятельству можно показать (см. [57], [58] и цитированную там литературу), что ДП $\sigma(\omega)(29)$ определяется формулой Кубо

$$
\sigma(\omega)=\int_{0}^{\infty} e^{i \omega t} f_{\sigma}(t) d t, \quad f_{\sigma}(t)=-\frac{i}{3 \hbar V}\left\langle\left[\hat{I}^{\beta}(t), \widehat{P}^{\beta}(0)\right]\right\rangle,
$$

а для величины $\varkappa(32)$ справедливо равенство (см. (18))

$$
\varkappa^{2}=4 \pi \sum_{a, b} Z_{a} Z_{b} e^{2} \Delta_{a b} .
$$

Здесь $\hat{I}^{\beta}(t)$ - оператор полного тока, $\widehat{P}^{\beta}=\int r_{\beta} \hat{\rho}_{z}(\mathbf{r}) d \mathbf{r}$ - оператор дипольного момента для зарядов, $\hat{\rho}_{z}(\mathbf{r})$ - оператор плотности заряда. Определение (40) для временно́й корреляционной функции $f_{\sigma}(t)$ следует понимать в термодинамическом пределе.

Если найденная величина $\Pi_{Z Z}(q, z)$ является аналитической функцией в верхней полуплоскости комплексных $z$, то эта функция может быть представлена в виде временно́го интеграла вида (35), (40). Тогда уже нет необходимости в реализации процедуры (37), а достаточно осуществить замену $z$ на $\omega$ [57]. Это утверждение легко проверить на примере поляризационного оператора для бесстолкновительной плазмы [50].

Рассмотрим случай взаимодействующих заряженных частиц. Воспользуемся неравенством $\varepsilon(q)>1$, которое является достаточным условием того, что ДП $\varepsilon(q, z)$ является аналитической функцией в верхней полуплоскости комплексных $z$ при соответствующем значении волнового вектора $q$ [59]. Согласно (32), (41) это неравенство выполняется в области малых волновых векторов $(q \rightarrow 0)$ при произвольных термодинамических параметрах КС. С учетом (38) приходим к выводу, что поляризационный оператор $\Pi_{Z Z}(q, z)$ также является аналитической функцией в верхней полуплоскости комплексных $z$ при малых значениях волнового вектора $q$. Следовательно, функция $\Pi_{Z Z}(q, \omega)(39)$ может быть представлена в виде [50]

$$
\Pi_{Z Z}(q, \omega)=\int_{0}^{\infty} d t e^{i \omega t} \varphi_{\Pi}(q, t), \quad \lim _{t \rightarrow \infty} \varphi_{\Pi}(q, t)=0,
$$

в области малых волновых векторов $q$ при произвольных термодинамических параметрах КС. При этом согласно (29)-(32), (39), (42) должны быть одновременно выполнены предельные соотношения

$$
\lim _{q \rightarrow 0} \int_{0}^{\infty} d t \varphi_{\Pi}(q, t)=-\frac{\varkappa^{2}}{4 \pi}, \quad \lim _{q \rightarrow 0} \frac{4 \pi}{q^{2}} \int_{0}^{\infty} d t e^{i \omega t} \varphi_{\Pi}(q, t)=1-\varepsilon(\omega) .
$$

Из (43) непосредственно следует, что при $\varkappa^{2} \neq 0$ функция $\varepsilon(\omega)$ должна иметь особенность при $\omega \rightarrow 0$, которая согласно (31) связана с ненулевой статической проводимостью $\sigma_{\text {st }}$.

Если статическая проводимость равна нулю, что отвечает состоянию истинного диэлектрика, то в соответствии с (43) величина $\varkappa^{2}$ должна обращаться в нуль:

$$
\sigma_{\mathrm{st}} \rightarrow 0 \quad \leftrightarrow \quad \varkappa \rightarrow 0
$$


Это означает, что в состоянии истинного диэлектрика неравенство (28) нарушается и $\lim _{q \rightarrow 0} \lim _{\omega \rightarrow 0} \varepsilon(q, \omega)=\lim _{\omega \rightarrow 0} \lim _{q \rightarrow 0} \varepsilon(q, \omega)=\varepsilon^{T}$, т. е. значение ДП $\varepsilon(q, \omega)$ не зависит от порядка предельных переходов $\omega \rightarrow 0, q \rightarrow 0$ и $q \rightarrow 0, \omega \rightarrow 0$, а величину $\varepsilon^{T}$ вполне естественно считать диэлектрической постоянной истинного диэлектрика. Как следует из (33), величина $\varepsilon^{T}$ характеризует изменение амплитуды внешнего поля в истинном диэлектрике при полном отсутствии экранирования (как эффекта уменьшения амплитуды при проникновении электростатического поля в вещество; подробнее см. [49], [57]).

Таким образом, при определении термодинамических параметров перехода в состояние истинного диэлектрика вместо условия $\sigma_{\mathrm{st}} \rightarrow 0$ для статической проводимости как кинетического коэффициента [45] может быть использовано условие для термодинамических функций (см. (41))

$$
\sum_{a, b} Z_{a} Z_{b} e^{2} \Delta_{a b} \rightarrow 0
$$

Это утверждение является строгим результатом для однородных и изотропных КС в нерелятивистском приближении [50].

\section{4. АНОМАЛИЯ ИЗОТЕРМИЧЕСКОЙ СЖИМАЕМОСТИ И СОСТОЯНИЕ ИСТИННОГО ДИЭЛЕКТРИКА}

С учетом представленных выше результатов перейдем к рассмотрению условий, при которых в $\mathrm{KC}$ может наблюдаться критическая опалесценция, связанная с аномальным поведением изотермической сжимаемости (3).

Прежде всего, обратим внимание, что из условия термодинамической устойчивости $K_{T}>0$ согласно (26) следует

$$
\Delta_{\mathrm{ee}} \Delta_{\mathrm{cc}}>\Delta_{\mathrm{ec}}^{2}
$$

В этом нетрудно убедиться, если учесть, что при использовании большого канонического распределения Гиббса имеют место равенства

$$
\begin{gathered}
k_{\mathrm{B}} T \Delta_{a b}=\frac{1}{V}\left\langle\delta \widehat{N}_{a} \delta \widehat{N}_{b}\right\rangle, \quad \delta \widehat{N}_{a}=\widehat{N}_{a}-\left\langle\widehat{N}_{a}\right\rangle \\
n_{\mathrm{c}}^{2} \Delta_{\mathrm{ee}}-2 n_{\mathrm{e}} n_{\mathrm{c}} \Delta_{\mathrm{ec}}+n^{2} \Delta_{\mathrm{cc}}=\frac{n_{\mathrm{c}}^{2}}{k_{\mathrm{B}} T} \frac{\left\langle\widehat{Z}^{2}\right\rangle}{V} \geqslant 0, \quad \widehat{Z}=\sum_{a} Z_{a} e \widehat{N}_{a} .
\end{gathered}
$$

В (48) учтено условие квазинейтральности (6). Из (47), (48), в частности, следует, что величина $\varkappa(41)$, определяющая радиус экранирования внешнего электростатического поля, равна

$$
\varkappa=\left(\frac{4 \pi}{k_{\mathrm{B}} T} \frac{\left\langle\widehat{Z}^{2}\right\rangle}{V}\right)^{1 / 2} .
$$

Соотношения (47)-(49) следует понимать в термодинамическом пределе.

Таким образом, выполнение предельного соотношения (3) для изотермической сжимаемости, приводящее к критической опалесценции, в двухкомпонентной КС согласно (26), (46)-(49) возможно в двух случаях [48], [49]: 
1) при одновременном выполнении предельных соотношений

$$
\Delta_{\text {eе }} \rightarrow \infty \quad \text { и } \quad \Delta_{\text {сс }} \rightarrow \infty ;
$$

2) при выполнении условия

$$
\varkappa^{2} \rightarrow 0
$$

Условия (50) соответствуют предельному соотношению для определения критических параметров фазового перехода газ-жидкость для модельной однокомпонентной системы с короткодействующим потенциалом межчастичного взаимодействия в соответствии с термодинамическим равенством

$$
-\frac{1}{V}\left(\frac{\partial V}{\partial P}\right)_{T, N}=\frac{1}{n}\left(\frac{\partial n}{\partial \mu}\right)_{T} .
$$

Тем самым определение критической точки в простой жидкости может быть записано как

$$
\left(\frac{\partial n}{\partial \mu}\right)_{T}=\frac{1}{k_{\mathrm{B}} T V}\langle\delta \widehat{N} \delta \widehat{N}\rangle \rightarrow \infty
$$

т. е. в критической точке квадрат флуктуаций полного числа частиц в заданном объеме стремится к бесконечности (см., например, [54]).

В свою очередь, предельное соотношение (2) для статического структурного фактора в простой жидкости может быть доказано только на основе большого канонического распределения Гиббса и имеет следующий вид (см., например, [7]):

$$
\lim _{q \rightarrow 0} S(q)=k_{\mathrm{B}} T\left(\frac{\partial n}{\partial \mu}\right)_{T}
$$

это соотношение переходит в (2) при использовании (52).

Отличие от простой (нейтральной) жидкости заключается в том, что вместо одного (см. (53)) имеются два предельных соотношения (50) для КС, которая изначально является двухкомпонентной системой. Таким образом, мы можем считать, что условия (50) соответствуют “обычной” критической точке фазового перехода газ-жидкость в двухкомпонентной КС.

Обратимся теперь к условию (51), возможность выполнения которого означает, что в двухкомпонентной КС имеется вторая критическая точка, отвечающая состоянию истинного диэлектрика с нулевой статической проводимостью (см. (44)). В этом смысле состояние истинного диэлектрика, как и сама критическая точка, является предельным состоянием, но в отличие от традиционного представления это состояние не связано непосредственно с нулевой температурой.

Существование второй критической точки в веществе, состоящем из одного химического элемента, косвенно подтверждается экспериментальными данными, причем подобная критическая точка, по-видимому, связана с фазовым переходом жидкость-жидкость (см., например, [46], [47], [51]). 
Проведенное выше рассмотрение имеет место, если условия (50) и (51) независимы. Нам остается рассмотреть случай, которому до настоящего времени не уделялось внимания, а именно выяснить, когда соотношение (51) справедливо при выполнении условий (50). Для этого введем две функции $\alpha\left(\mu_{\mathrm{e}}, \mu_{\mathrm{c}}, T\right)$ и $\gamma\left(\mu_{\mathrm{e}}, \mu_{\mathrm{c}}, T\right)$ :

$$
\alpha\left(\mu_{\mathrm{e}}, \mu_{\mathrm{c}}, T\right) \equiv \frac{\Delta_{\mathrm{cc}}}{\Delta_{\mathrm{ee}}}, \quad \gamma\left(\mu_{\mathrm{e}}, \mu_{\mathrm{c}}, T\right) \equiv \frac{\Delta_{\mathrm{ec}}}{\Delta_{\mathrm{ee}}} .
$$

Тогда согласно $(20),(27),(46)$ изотермическая сжимаемость двухкомпонентной КС равна

$$
K_{T}=\Delta_{\mathrm{ee}} \frac{\alpha-\gamma^{2}}{n_{\mathrm{c}}^{2}\left(1-2 Z_{\mathrm{c}} \gamma+Z_{\mathrm{c}}^{2} \alpha\right)}=\frac{\Delta_{\mathrm{ee}}}{n_{\mathrm{c}}^{2}} \frac{\alpha-\gamma^{2}}{\left\{\left(1-Z_{\mathrm{c}} \gamma\right)^{2}+Z_{\mathrm{c}}^{2}\left(\alpha-\gamma^{2}\right)\right\}}, \quad \alpha>\gamma^{2} .
$$

В критической точке $\left(K_{T} \rightarrow \infty\right)$ при выполнении условий $(50)$ с учетом $(55),(56)$ возможны два варианта:

$$
\begin{array}{ll}
\text { I }: & \alpha-\gamma^{2} \rightarrow \text { const } \neq 0 ; \\
\text { II }: & \alpha-\gamma^{2} \rightarrow 0 .
\end{array}
$$

Как следует из проведенного выше рассмотрения, вариант I соответствует обычной критической точке фазового перехода газ-жидкость. При реализации варианта II возможны два случая. Первый случай, когда в критической точке

$$
\frac{\alpha-\gamma^{2}}{\left(1-Z_{\mathrm{c}} \gamma\right)^{2}} \rightarrow 0
$$

не представляет интереса для проводимого рассмотрения в силу возникающей неопределенности вида $\infty \cdot 0$. В случае же, когда

$$
\frac{\alpha-\gamma^{2}}{\left(1-Z_{\mathrm{c}} \gamma\right)^{2}} \rightarrow \text { const } \neq 0
$$

как нетрудно убедиться, имеет место соотношение (51) для истинного диэлектрика.

\section{5. ЗАКЛЮЧЕНИЕ}

Таким образом, мы приходим к выводу, что в двухкомпонентной КC, состоящей из электронов и ядер одного сорта, явление критической опалесценции может наблюдаться не только в критической точке, отвечающей фазовому переходу газ-жидкость, но и при термодинамических параметрах в окрестности состояния истинного диэлектрика с нулевой статической проводимостью. С учетом имеющихся экспериментальных данных можно заключить, что состояние истинного диэлектрика является предельным состоянием фазового перехода жидкость-жидкость, который сопровождается резким изменением электропроводности вещества. Установлено, что это состояние может соответствовать условию обращения в бесконечность квадрата флуктуаций как полного числа электронов, так и полного числа ядер для рассматриваемой КС в заданном объеме. При этом для исследования состояния истинного диэлектрика могут быть использованы эксперименты по рассеянию не только электромагнитного излучения, но и нейтронов. 
Благодарности. Работа выполнена при поддержке Российского фонда фундаментальных исследований (грант № 12-08-00600-а).

\section{Список литературы}

[1] B. Chu, Laser Light Scattering, Akademic Press, New York, 1991.

[2] D. I. Ivanov, Critical Behavior of Non-Ideal Systems, Wiley-VHC, Vienheim, 2008.

[3] M. V. Smoluchowski, Ann. Phys., 330:2 (1908), 205-226.

[4] A. Einstein, Ann. Phys., 338:16 (1910), 1275-1298.

[5] P. Résibois, M. De Leener, Classical Kinetic Theory of Fluids, Wiley, New York, 1977.

[6] L. S. Ornstein, F. Zernike, Proc. Acad. Sci. Amsterdam, 17 (1914), 793-806.

[7] R. Balescu, Equilibrium and Nonequilibrium Statistical Mechanics, Wiley, New York, 1975.

[8] J. E. Thomas, P. W. Schmidt, J. Chem. Phys., 39:10 (1963), 2506-2515.

[9] M. A. Anisimov, Critical Phenomena in Liquids and Liquid Crystals, Gordon and Breach, Philadelphia, 1991.

[10] G. A. Martynov, Fundamental Theory of Liquids. Method of Distribution Functions, Adam Hilger, Bristol, 1992.

[11] Г. А. Мартынов, УФН, 169:6 (1999), 595-624.

[12] L. D. Landau, Phys. Z. Sowjetunion, 11 (1937), 26-35.

[13] E. A. Guggenheim, J. Chem. Phys., 13:7 (1945), 253-261.

[14] X.-G. Wen, Phys. Rev. B, 40:10 (1989), 7387-7390.

[15] S.S. Jahromi, S. F. Masoudi, M. Kargarian, K. P. Schmidt, Phys. Rev. B , 88:21 (2013), 214411, 8 pp., arXiv: 1308.1407.

[16] L. Onsager, Phys. Rev., 65:3-4 (1944), 117-149.

[17] E. Ising, Z. Phys., 31:1 (1925), 253-258.

[18] B. Widom, J. Chem. Phys., 41:6 (1964), 1633-1634; 43:11 (1965), 3892-3897; 3898-3904.

[19] L.P. Kadanoff, Physics, 2:6 (1966), 263-272; Nuovo Cimento B Ser. 10, 44:2 (1966), 276-305.

[20] K. G. Wilson, Phys. Rev. B, 4:9 (1971), 3174-3183; 3184-3205.

[21] M. E. Fisher, Rev. Modern Phys., 70:2 (1998), 653-681.

[22] H. E. Stanley, Rev. Modern Phys., 71:2 (1999), S358-S366.

[23] M. Barmatz, I. Hahn, J. A. Lipa, R. V. Duncan, Rev. Modern Phys., 79:1 (2007), 1-52.

[24] A. Parola, L. Reatto, Adv. Phys., 44:3 (1995), 211-298.

[25] Г. А. Мартынов, ТМФ, 119:3 (1999), 498-516; 156:3 (2008), 454-464.

[26] G. A. Martynov, J. Chem. Phys., 129:24 (2008), 244509; Phys. Rev. E, 79:3 (2009), 031119, 9 pp.

[27] R. Evans, J. R. Henderson, J. Phys.: Cond. Matt., 21:47 (2009), 474220, 11 pp.

[28] M.E. Fisher, J. Phys.: Cond. Matt., 8:47 (1996), 9103-9110.

[29] А. А. Ликальтер, УФН, 43:7 (2000), 831-854.

[30] W.-D. Kraeft, D. Kremp, W. Ebeling, G. Röpke, Quantum Statistics of Charged Particle Systems, Plenum, New York, 1986.

[31] D. Pines, P. Noziéres, The Theory of Quantum Liquids, Benjamin, New York, 1966.

[32] V.B. Bobrov, S. A. Trigger, Phys. Lett. A, 375:16 (2011), 1716-1719.

[33] V. B. Bobrov, J. Phys.: Cond. Matt., 2:13 (1990), 3115-3118.

[34] В. Б. Бобров, Н. И. Ключников, С. А. Тригер, ТМФ, 89:2 (1991), 263-277.

[35] V.B. Bobrov, N. I. Klyuchnikov, S. A. Trigger, Physica A: Statist. Mech. Appl., 181:1-2 (1992), 156-172.

[36] V. B. Bobrov, I. M. Sokolov, S. A. Trigger, Phys. Plasmas, 19:6 (2012), 062101, 8 pp. 
[37] Л. Д. Ландау, Е. М. Лифшиц, Теоретическая физика, т. 3: Квантовая механика. Нерелятивистская теория, Наука, М., 1974.

[38] В. Б. Бобров, ТМФ, 178:3 (2014), 433-448.

[39] S. Dietrich, A. Haase, Phys. Rep., 260:1-2 (1995), 1-138.

[40] J. Chihara, J. Phys.: Cond. Matt., 12:3 (2000), 231-248.

[41] V. B. Bobrov, S. A. Trigger, S. N. Skovorod'ko, Europhys. Lett., 92:1 (2010), 13001, 5 pp.

[42] F. Evers, A. D. Mirlin, Rev. Modern Phys., 80:4 (2008), 1355-1417.

[43] В. Ф. Гантмахер, В. Т. Долгополов, УФН, $\mathbf{5 1 : 1 ~ ( 2 0 0 8 ) , ~ 3 - 2 4 . ~}$

[44] В. Б. Бобров, В. Я. Менделеев, С. А. Тригер, Г. ван Хейст, П. Шрам, ТВT, 51:4 (2013), 511-519.

[45] R. Redmer, B. Holst, F. Hensel (eds.), Metal-to-Nonmetal Transitions, Springer, Berlin, 2010.

[46] N. Subramanian, A. F. Goncharov, V. V. Struzhkin, M. Somayazulu, R. J. Hemley, Proc. Natl. Acad. Sci. USA, 108:15 (2011), 6014-6019.

[47] V. Dzyabura, M. Zaghoo, I. F. Silvera, Proc. Natl. Acad. Sci. USA, 110:20 (2013), 8040-8044.

[48] V. B. Bobrov, S. A. Trigger, J. Phys. A: Math. Theor., 43:36 (2010), 365002, 11 pp.

[49] В. Б. Бобров, С. А. Тригер, ТВТ, 49:4 (2011), 513-523.

[50] V. B. Bobrov, Phys. Rev. E, 86:2 (2012), 026401, 4 pp.

[51] V. B. Bobrov, S. A. Trigger, A. G. Zagorodny, Europhys. Lett., 101:1 (2013), 16002, 5 pp.

[52] А. А. Абрикосов, Л. П. Горьков, И. Е. Дзялошинский, Методы квантовой теории поля в статистической физике, Физматгиз, М., 1962.

[53] В.П. Силин, А. А. Рухадзе, Электромагнитные свойства плазмы и плазмоподобных сред, Госатомиздат, М., 1961.

[54] Л. Д. Ландау, Е. М. Лифшиц, Теоретическая физика, т. 5: Статистическая физика. Часть 1, Наука, М., 1976.

[55] Е. М. Лифшиц, Л. П. Питаевский, Теоретическал физика, т. 10: Физическал кинетика, Наука, М., 1979.

[56] Д. Н. Зубарев, Неравновесная статистическая термодинамика, Наука, М., 1971.

[57] V. B. Bobrov, S. A. Trigger, A. G. Zagorodny, Phys. Lett. A, 375:2 (2010), 84-87.

[58] V.B. Bobrov, S. A. Trigger, G. J. F. van Heijst, P. P. J. M. Schram, Europhys. Lett., 90:1 (2010), 10003, 4 pp.

[59] O. V. Dolgov, D. A. Kirzhnitz, E. G. Maksimov, Rev. Modern Phys., 53:1 (1981), 81-93. 